\title{
Gastrointestinal failure score in children with traumatic brain injury
}

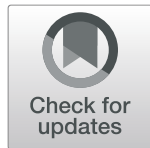

\author{
Ying Zhou, Weifeng Lu and Weibing Tang
}

\begin{abstract}
Background: To review the value of the gastrointestinal failure (GIF) score in children with different degrees of traumatic brain injury (TBI) by analyzing the correlation between outcome and gastrointestinal function.

Methods: A total of 165 children with TBI who were diagnosed and treated in the surgical intensive care unit (SICU) for longer than $72 \mathrm{~h}$ between August 2017 and September 2019 were analyzed. Admission parameters included sex, age, Glasgow Coma Scale (GCS) score, body mass index (BMI), leukocyte count, C-reactive protein (CRP), hemoglobin $(\mathrm{Hb})$, hematocrit (Hct), blood glucose, lactic acid, procalcitonin (PCT), albumin, plasma osmotic pressure, prothrombin time (PT) and activated partial thromboplastin time (APTT). To predict outcomes, the Pediatric Sequential Organ Failure Assessment (SOFA) score, Pediatric Clinical Illness Score (PCIS), and mean GIF score for the first three days were combined.
\end{abstract}

Results: The percentage of patients with gastrointestinal dysfunction on the first day was $78.8 \%$. Food intolerance (FI) and intra-abdominal hypertension (IAH) developed in 36.4 and $21.8 \%$ of the patients, respectively. The GIF score and mean GIF score for the first three days were significantly different between children with different degrees of TBI $(P<0.05)$; these scores were also significantly different between patients who died and those who survived $(P<$ 0.05). The mean GIF score for the first three days was identified as an independent risk factor for mortality (odds ratio $>1,95 \%$ confidence interval $=1.457$ to $16.016, P<0.01$ ), as was the PCIS. Receiver operating characteristic (ROC) curve analysis suggested that the mean GIF score for the first three days had the same calibrating power as the PCIS in discriminating the risk of death of children.

Conclusions: The incidence of gastrointestinal dysfunction in children with TBI is high. The GIF score has the ability to reflect the status of the gastrointestinal system. The mean GIF score for the first three days has high prognostic value for ICU mortality in the SICU.

Keywords: Gastrointestinal failure (GIF) score, Traumatic brain injury, Children

\section{Background}

Traumatic brain injury (TBI) has the highest mortality and morbidity of all types of trauma and represents a serious threat to the life and physical health of children. According to statistics, the incidence of TBI in European and American countries is as high as $150-200 / 100,000 /$ year, while the incidence is approximately $100-150 / 100$,

\footnotetext{
*Correspondence: twbcn@njmu.edu.cn

Department of Pediatric Surgery, Children's Hospital of Nanjing Medical University, 72 Guangzhou Road, Jiangsu Province 210008 Nanjing, China
}

000/year in China [1, 2]. Due to their young age, low crisis awareness and poor self-protection ability, severe TBIs resulting in a Glasgow Coma Scale (GCS) score of less than 8 are very common in children. Children with such TBIs have a mortality rate of $20 \%$ and a severe disability rate of $>50 \%$ [3] and require monitoring and treatment in intensive care units (ICUs). Children in ICUs with gastrointestinal bleeding, dysfunction or failure often endure prolonged hospital stays and have increased mortality [4]. However, objectively evaluating gastrointestinal dysfunction is difficult because

(c) The Author(s). 2021 Open Access This article is licensed under a Creative Commons Attribution 4.0 International License, which permits use, sharing, adaptation, distribution and reproduction in any medium or format, as long as you give appropriate credit to the original author(s) and the source, provide a link to the Creative Commons licence, and indicate if changes were made. The images or other third party material in this article are included in the article's Creative Commons licence, unless indicated otherwise in a credit line to the material. If material is not included in the article's Creative Commons licence and your intended use is not permitted by statutory regulation or exceeds the permitted use, you will need to obtain permission directly from the copyright holder. To view a copy of this licence, visit http://creativecommons.org/licenses/by/4.0/. The Creative Commons Public Domain Dedication waiver (http://creativecommons.org/publicdomain/zero/1.0/) applies to the data made available in this article, unless otherwise stated in a credit line to the data. 
quantitative standards to classify severity are lacking [5, 6]. Few of the various existing scoring systems for determining the severity of disease in pediatric patients include an evaluation of gastrointestinal function [7]. The gastrointestinal failure (GIF) score serves as an objective indicator used to evaluate gastrointestinal dysfunction [8]. This study collected the clinical data of children with TBI admitted to the surgical intensive care unit (SICU) in our hospital, and GIF scores were estimated. The purpose of this study was to reveal the importance of gastrointestinal dysfunction and its impact on the prognosis of children with TBI and to provide reliable evidence for the evaluation of gastrointestinal function in children with TBI.

\section{Methods}

\section{Ethics}

Written informed consent was obtained from the patients and/or their parents, and this project was approved by the ethics committee of Children's Hospital of Nanjing Medical University (No. 202001004-1).

\section{Clinical information}

We conducted a prospective observational study at Children's Hospital of Nanjing Medical University in China. We included patients admitted from August 2017 to Sept 2019 who met the following criteria: (a) age 3 months to 13 years and 8 months; (b) admission with TBI (defined as severe with GCS score from 3 to 8 , moderate with GCS score from 9 to 12 and mild with GCS score from 13 to 15 ) within 24 h of injury; (c) confirmation of intracranial injury on head CT as brain edema, subdural hemorrhage and intracranial hemorrhage; (d) no treatment with sedatives; and (e) no history of previous intracranial or gastrointestinal disease. We excluded patients with (a) neonatal age ( $<28$ days); (b) TBI combined with primary gastrointestinal injury; (c) admission to the hospital more than $24 \mathrm{~h}$ after injury; (d) hospital stay shorter than $72 \mathrm{~h}$; and (e) TBI combined with organ failure, serious metabolic disorders, and other basic diseases.

The clinical parameters that were collected on admission included sex, age, body mass index (BMI), GCS score, leukocyte count, C-reactive protein (CRP) level, hemoglobin $(\mathrm{Hb})$, hematocrit $(\mathrm{Hct})$, blood glucose (Glu), lactic acid (Lac), procalcitonin (PCT), albumin (ALB), plasma osmotic pressure (POP), prothrombin time (PT) and activated partial thromboplastin time (APTT). The Sequential Organ Failure Assessment (SOFA) score and Pediatric Clinical Illness Score (PCIS) were recorded daily. The gastrointestinal function of the patients was evaluated daily by the GIF score as follows [8]: $0=$ normal gastrointestinal function; $1=$ enteral feeding with under $50 \%$ of the calculated need; $2=$ food intolerance
(FI) or intra-abdominal hypertension (IAH); $3=\mathrm{FI}$ and IAH; and $4=$ abdominal compartment syndrome (ACS). The SOFA scores, PCIS and mean GIF scores for the first three days were combined to predict outcome. The primary outcome parameter was ICU mortality.

A few caveats should be noted. Enteral feeding was provided as early as possible. Stress ulcers were diagnosed if dark blood fluid was found in gastrointestinal decompression tubes or gastric and duodenal mucosa erosion and ulcers were observed by gastroscopy. The criteria for FI diagnosis were failed enteral feeding or vomiting after eating more than three times a day, gastric residual volumes exceeding $50 \%$ of the feeding volume, and intestinal obstruction, severe diarrhea, or bloating that could not be resolved within $24 \mathrm{~h}$. If FI developed, intra-abdominal pressure (IAP) was measured with an empty bladder in the supine position using the closed-loop system repeated-measurements technique [9]. IAP was measured at least twice per day when normal. When the IAP was higher than $12 \mathrm{mmHg}$, it was measured four times per day at different points. IAH was defined as a persistent IAP of $12 \mathrm{mmHg}$ or higher. ACS was defined as a persistent IAP of $20 \mathrm{mmHg}$ or greater accompanied by new organ failure. The mean and maximum IAP values were documented daily, and the mean value was used to calculate the daily GIF score.

\section{Statistical analysis}

SPSS 19.0 (Professional Edition) was used for data analysis. The data are presented as the mean \pm standard $(' x \pm s)$ unless stated otherwise. Differences between two groups were evaluated by the two-sample $\mathrm{T}$ test for continuous variables and by the chi-square test (or Fisher's exact probability) for categorical variables. One-way ANOVA was used to compare multiple means. GIF scores for the first three days were calculated as the mean individual score for three days for every child. Risk factors for ICU mortality were identified by univariate analyses of admission parameters, and parameters with $p<0.1$ were entered into a multiple logistic regression model to identify independent risk factors. The collinearity of the parameters in the regression was checked. Receiver operating characteristic (ROC) curves were used to determine the likelihood ratios for the abilities of the GIF score, SOFA score and PCIS to predict ICU mortality. $P<0.05$ was considered statistically significant.

\section{Results}

This study included 165 children (103 boys (62.4\%) and 62 girls $(37.6 \%)$ ) with an average age of 4 years and 11 months. There were 92 cases of severe, 23 cases of moderate and 50 cases of mild craniocerebral injury; the incidence rate of stress ulcers was much higher in the severe 
group than in the moderate and mild groups $(85.9 \%$, $4.3 \%$, and $0 \%$, respectively, $p<0.05$ ), as was the incidence rate of secondary gastrointestinal dysfunction (100\%, $82.6 \%$, and $38 \%$, respectively). The GIF score on the first day and the mean GIF score for the first three days differed significantly among the three groups $(p<$ 0.05, Table 1).

A total of 130 patients $(78.8 \%)$ had gastrointestinal dysfunction on the first day of hospital admission, including 34 with insufficient feeding (20.6\%), 60 with FI $(36.4 \%)$, and 36 with IAH or ACS (21.8\%). Eighty children had TBI with stress ulcers $(48.5 \%)$. The children were divided into two groups based on whether FI occurred on the first day of admission. Sixty-nine children (41.8\%) had a GIF score $<2$ on the first day, and 96 children $(58.2 \%)$ had a GIF score $\geq 2$. Significant differences were observed in GCS and GIF scores on the first day, the mean GIF scores for the first three days of admission, SOFA scores, and PCISs between the two groups $(p<0.05$, Table 2$)$.

\section{Multivariate regression and receiver operating characteristic (ROC) curve analyses}

Lab parameters except C-reactive protein, hematocrit, procalcitonin and plasma osmotic pressure were significantly different between the deceased and survival groups $(p<0.05)$. There was no collinearity among the parameters in the regression $(\mathrm{VIF}<5)$. Binary multivariate logistic regression analysis was performed using the SOFA score, PCIS, GIF score on the first day of admission, and mean GIF score for the first three days to establish a risk of death prediction model. The overall accuracy rate of this model in predicting death was $90.9 \%$. The PCIS and mean GIF score for the first three days were independent risk factors for death (odds ratio
$(\mathrm{OR})>1$, Table 3). ROC analysis showed that the PCIS and the mean GIF score for the first three days had good predictive ability for the death of children with TBI (Fig. 1).

\section{Discussion}

In this study, 135 children with TBI (78.8\%) had gastrointestinal dysfunction on the first day of admission, suggesting that gastrointestinal dysfunction in children with TBI is very common. The incidence of stress ulcers in children with TBI was $48.5 \%$ and gradually increased with TBI severity, with a rate of $85.9 \%$ in severe cases, suggesting that the risk of gastrointestinal mucosal ischemia is high, which is consistent with previous reports in adult TBI patients [10]. The gastrointestinal tract is the only system that is jointly controlled by the central nervous system, enteric nerves, and autonomic nerves; thus, the complex neuroendocrine network named the brain-gut axis plays an important role in regulating gastrointestinal function. After the central nervous system is damaged, various pathways in the brain-gut axis are blocked, and many brain-gut peptides are secreted abnormally. These pathways and peptides cannot transmit information normally or stimulate gastrointestinal motility [11-13]. The gastrointestinal mucosa is in a state of hypoperfusion, resulting in the spread of focal small ulcers, which usually manifest as stress ulcers. In addition, gastrointestinal hormone levels and secretion are disordered, and intestinal flora are imbalanced. Feedback from the gastrointestinal tract to the central nervous system is abnormal, causing gastrointestinal dysfunction [14-16].

Approximately one third of the children developed FI on the first day of ICU admission. These children were significantly more ill (lower GCS scores and higher SOFA scores and PCISs) and exhibited greater morality

Table 1 Admission and outcome parameters for different degrees

\begin{tabular}{lclll}
\hline Parameters & Severe & Moderate & Mild & p \\
\hline Number (\%) & $92(55.8)$ & $23(13.9)$ & $50(30.3)$ & \\
Age & $4.70 \pm 3.57$ & $5.08 \pm 4.63$ & $5.05 \pm 4.03$ & $\mathrm{~F}=0.18, p=0.84$ \\
BMI $\left(\mathrm{kg} / \mathrm{m}^{2}\right)$ & $16.46 \pm 2.99$ & $18.08 \pm 5.06$ & $16.81 \pm 2.96$ & $\mathrm{~F}=2.17, p=0.12$ \\
Stress ulcer (\%) & 85.90 & 4.30 & 0.00 & $X^{2}=116.48^{2}, p<0.05$ \\
GSC & $4.96 \pm 1.82$ & $10.83 \pm 1.07$ & $13.50 \pm 0.51$ & $\mathrm{~F}=606.14, p<0.05$ \\
Incubation (\%) & $87(94.57)$ & $2(8.70)$ & $0(0)$ & $X^{2}=138.62^{2}, p<0.05$ \\
SOFA score & $7.86 \pm 2.77$ & $4.00 \pm 1.81$ & $2.10 \pm 1.18$ & $\mathrm{~F}=110.03, p<0.05$ \\
PCIS score & $73.91 \pm 10.36$ & $91.48 \pm 7.70$ & $95.80 \pm 3.66$ & $\mathrm{~F}=120.43, p<0.05$ \\
First day GIF score & $2.28 \pm 0.56$ & $0.96 \pm 0.56$ & $0.60 \pm 0.95$ & $\mathrm{~F}=104.16, p<0.05$ \\
Mean GIF score for the first three days & $2.02 \pm 0.44$ & $0.57 \pm 0.38$ & $0.42 \pm 0.75$ & $\mathrm{~F}=165.62, p<0.05$ \\
Mortality (\%) & 43.5 & 0 & 0 & $X^{2}=41.90^{2}, p<0.05$ \\
\hline
\end{tabular}

Unit of measure provided in parentheses 
Table 2 Admission and outcome parameters for GIF score $<2$ and GIF score $\geq 2$

\begin{tabular}{llcc}
\hline Parameters & GIF score $<\mathbf{2}$ & GIF score $\geq \mathbf{2}$ & $\mathbf{p}$ \\
\hline Number(\%) & $69(41.8)$ & $96(58.2)$ & \\
Age & $4.82 \pm 4.23$ & $4.96 \pm 3.64$ & $\mathrm{t}=0.23, \mathrm{p}=0.82$ \\
BMI $\left(\mathrm{kg} / \mathrm{m}^{2}\right)$ & $17.11 \pm 3.37$ & $16.56 \pm 3.36$ & $\mathrm{t}=-1.03, \mathrm{p}=0.30$ \\
GCS & $12.29 \pm 2.00$ & $5.54 \pm 2.83$ & $\mathrm{t}=17.91, p<0.05$ \\
First day GIF score & $0.49 \pm 0.50$ & $2.38 \pm 0.49$ & $\mathrm{t}=-24.02, p<0.05$ \\
Mean GIF score for the first three days & $0.31 \pm 0.36$ & $2.07 \pm 0.38$ & $\mathrm{t}=-29.86, p<0.05$ \\
SOFA score & $2.87 \pm 1.80$ & $7.52 \pm 3.06$ & $\mathrm{t}=-12.23, p<0.05$ \\
PCIS score & $94.09 \pm 6.17$ & $75.02 \pm 11.24$ & $\mathrm{t}=13.96, p<0.05$ \\
Mortality $(\%)$ & 0 & 41.67 & $x^{2}=37.95^{2}, p<0.05$ \\
\hline
\end{tabular}

Unit of measure provided in parentheses

than those without FI. The prevalence of FI was lower than that in previous studies of adult critically ill patients $[17,18]$. The reasons for this discrepancy are not clear, but we speculate that the following factors may be involved. (1) The included children did not have primary gastrointestinal injury. Compared with elderly critically ill patients with cardiovascular disease or diabetes, the children's organ function was basically normal. (2) Children's nervous systems are still developing; therefore, the stress response may not be completely elicited, and the abnormal release of brain-gut peptides is weakened [19]. (3) The gastrointestinal system of children can adapt to changes in the structure and function of neuronal circuits, and mucosal repair and functional reconstruction mechanisms are stronger in children than in adults [20]. Although we defined FI using objective measurements for greater precision, FI is a subjective variable and a universally used clinical characteristic covering the entire spectrum of gastrointestinal symptoms. FI allows a functional assessment with some clinical relevance [21], as shown in our study. IAH did not occur as frequently as FI in our study, and ACS occurred less often. References to adult evaluation criteria are of somewhat limited value. Further studies are needed to standardize the evaluation criteria.

The incidence of secondary gastrointestinal dysfunction increased with injury severity, and the GIF score on the first day and the mean GIF score for the first three days differed significantly among the severe, moderate and mild groups. The intestinal mucosa morphology was found to change within a short time after trauma, including epithelial cell detachment and apoptosis, rupture of the villi, edema of interstitial tissue and the lamina propria, interruption of tight junctions, etc., and the mucosal barrier was observed to have lost its protective function [11]. Additionally, the intestinal flora was completely disordered within a few hours after injury, and the microbial composition and relative abundance changed significantly. The number of beneficial microbiota decreased, while pathogenic flora, which showed relatively increased invasiveness and virulence, dominated the intestinal tract, and the diversity and stability of the microbial ecological system were destroyed. More severe trauma corresponded to worse dysbiosis and a greater effect on gastrointestinal function, leading to a high GIF score. The infection risk and the mortality rate of these pediatric patients were substantially increased [12].

The comparison of the relevant clinical indicators in the deceased and survival groups indicated that the GIF score on the first day and the mean GIF score for the first three days were significantly higher in the deceased group than in the survival group, as were the SOFA scores and PCISs. Multivariate logistic regression analysis suggested that a high mean GIF score for the first three days was closely related to mortality as an independent risk factor. The GIF score reflects

Table 3 Logistic regression analysis for different scores

\begin{tabular}{|c|c|c|c|c|c|c|c|c|}
\hline & \multirow[t]{2}{*}{ B } & \multirow[t]{2}{*}{ S.E, } & \multirow[t]{2}{*}{ Wals } & \multirow[t]{2}{*}{ df } & \multirow[t]{2}{*}{ Sig. } & \multirow{2}{*}{$\begin{array}{l}\text { Exp } \\
\text { (B) }\end{array}$} & \multicolumn{2}{|l|}{$95 \%$ C.I } \\
\hline & & & & & & & Lower limit & upper limit \\
\hline SOFA score & -0.14 & 0.17 & 0.70 & 1 & 0.40 & 0.87 & 0.63 & 1.20 \\
\hline PCIS & 0.25 & 0.06 & 15.64 & 1 & 0.00 & 1.29 & 1.14 & 1.46 \\
\hline First day GIF score & -0.66 & 0.68 & 0.94 & 1 & 0.33 & 0.51 & 0.14 & 1.97 \\
\hline Mean GIF score for the first three days & 1.58 & 0.61 & 6.63 & 1 & 0.01 & 4.83 & 1.46 & 16.02 \\
\hline constant & -17.96 & 5.87 & 9.37 & 1 & 0.00 & 0.00 & & \\
\hline
\end{tabular}




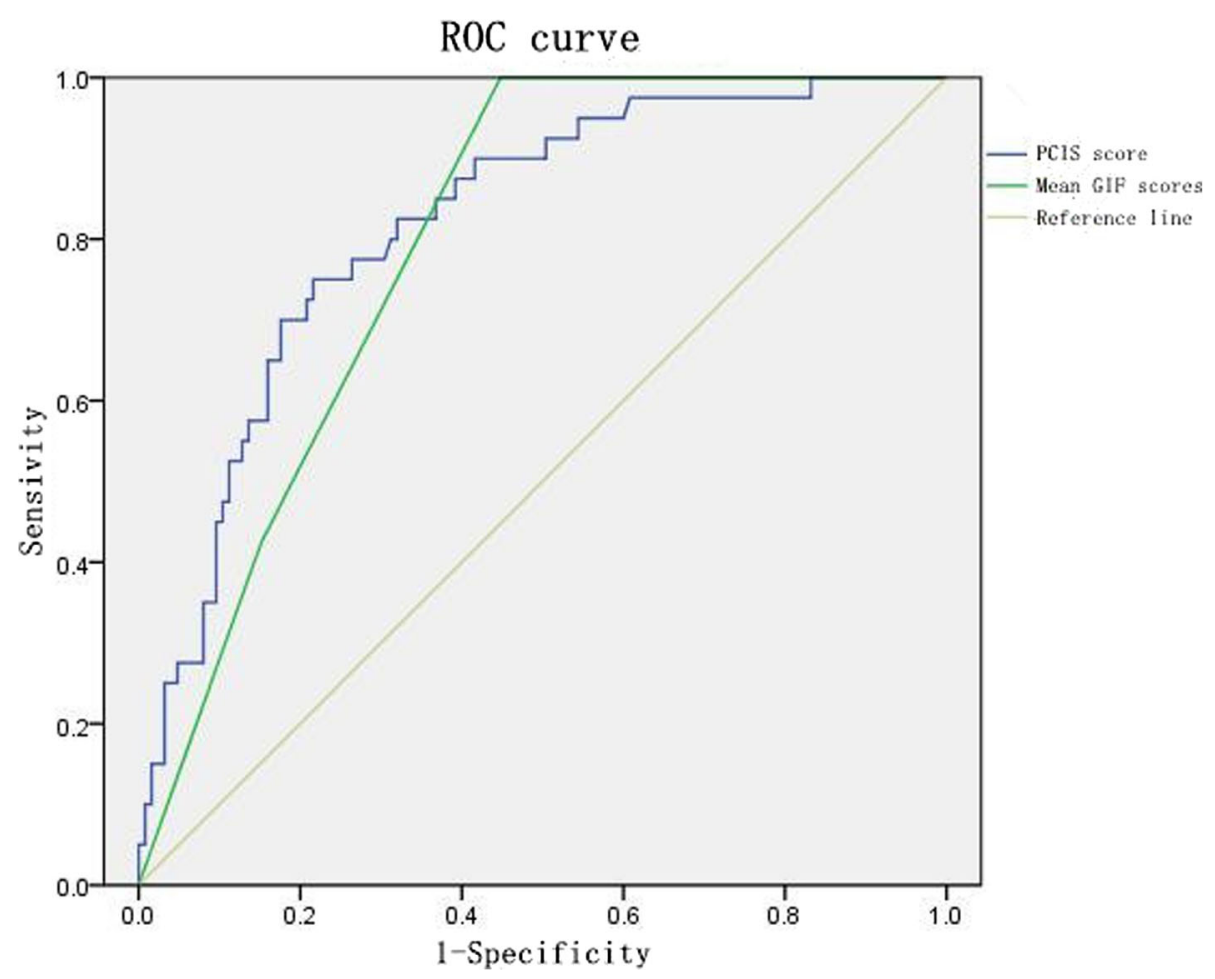

Fig. 1 ROC curves for the PCIS and mean GIF score for the first three days

gastrointestinal function, which can be classified into different levels, similar to other scoring systems for organ function failure. The clinical value and high reliability of the GIF score for predicting outcomes have been verified in intensive care patients and in digestive system diseases [10, 16, 22]. Although the GIF score can be used as an independent risk factor for predicting the risk of death in critically ill patients [23], it focuses on gastrointestinal function at the time of injury. However, the condition of a child develops and changes during hospitalization. The reliability of the GIF score on the first day was relatively low, and its role in predicting death during the entire ICU stay is limited [24]. The mean GIF score for the first three days can be used to dynamically observe and assess changes in gastrointestinal dysfunction during peak disease development, providing better continuity. Reintam et al. [8] found that the mean GIF score for the first three days was more important in predicting death than the GIF score on the first day. In this study, the mean GIF score for the first three days but not the GIF score on the first day was considered an independent risk factor. The reliability of the latter score was relatively low, suggesting that although the GIF score can be used as an objective indicator, the effectiveness and accuracy of dynamic observation and scoring are even higher. The mean GIF score for the first three days was better than the GIF score on the first day for evaluating the gastrointestinal function of children with TBI. One limitation of this study is that the SOFA score may be inapplicable to young infants and toddlers in terms of the items assessed [25-27]. The PCIS fully integrates the physiological and morbidity characteristics of children at different ages and was introduced to predict the risk of death $[28,29]$. In this study, the mean GIF score for the first three days had a predictive ability for death comparable to that of the PCIS. Both had good predictive abilities for the risk of death, again confirming the clinical significance of the GIF score in diagnosing gastrointestinal dysfunction in children with TBI and further emphasizing the importance of continuous monitoring and dynamic observation of the gastrointestinal status of children at different time points. Organ dysfunction in critically ill patients should be scored dynamically [30].

\section{Conclusions}

In summary, the incidence rate of gastrointestinal dysfunction in children with TBI is high. The GIF score can accurately classify and objectively assess gastrointestinal status. A high GIF score is significantly correlated with ICU mortality. As an independent risk factor, the mean GIF score for the first three days has higher value for predicting ICU mortality than the GIF score on the first day; this result can provide guidance for the clinical evaluation and treatment of gastrointestinal dysfunction in children with TBI. 


\section{Abbreviations}

TBI: Traumatic brain injury; SICU: Surgical intensive care unit; GCS: Glasgow Coma Scale; BMI: Body mass index; CRP: C-reactive protein; Hb: Hemoglobin; Hct: Hematocrit; PCT: Procalcitonin; PT: Prothrombin time; APTT: Activated partial thromboplastin time; SOFA: Sequential Organ Failure Assessment; PCIS: Pediatric Clinical IIIness Score; GIF: Gastrointestinal failure; FI: Food intolerance; IAH: Intra-abdominal hypertension; ACS: Abdominal compartment syndrome; IAP: Intra-abdominal pressure; ROC: Receiver operating characteristic

\section{Acknowledgements}

Not applicable.

\section{Conflict of interest}

Ying Zhou and the coauthors have no conflicts of interest.

\section{Authors' contributions}

YZ: Conceptualization, Methodology, Formal analysis, Data curation, WritingOriginal draft preparation, Writing- Reviewing and Editing. WL: Software, Validation. WT: Resources, Reviewing and Editing, and Supervision. All authors have read and approved the manuscript.

\section{Funding}

Not applicable.

\section{Availability of data and materials}

All data can be found in the medical records system of Children's Hospital of Nanjing Medical University. Since each piece of data corresponds to an identified patient, the details are inconvenient to disclose

\section{Declarations}

\section{Ethics approval and consent to participate}

Written informed consent was obtained from the patients and/or their parents, and this project was approved by the ethics committee of Children's Hospital of Nanjing Medical University (No. 202001004-1).

\section{Consent for publication}

Not applicable.

\section{Competing interests}

The authors declare that they have no competing interests.

Received: 19 June 2020 Accepted: 19 April 2021

Published online: 04 May 2021

\section{References}

1. Hackenberg K, Unterberg A. [Traumatic brain injury]. Nervenarzt. 2016;87(2): 203-14. quiz 215 - 206.

2. Maas Al, Stocchetti N, Bullock R. Moderate and severe traumatic brain injury in adults. Lancet Neurol. 2008;7(8):728-41.

3. Zhang LM, Li R, Sun WB, Wang XP, Qi MM, Bai Y, Bai J, Zheng WC. LowDose, Early Fresh Frozen Plasma Transfusion Therapy After Severe Trauma Brain Injury: A Clinical, Prospective, Randomized, Controlled Study. World Neurosurg. 2019;132:e21-7

4. Marker S, Perner A, Wetterslev J, Krag M, Lange T, Wise MP, Borthwick M, Bendel S, Keus F, Guttormsen AB, et al. Pantoprazole prophylaxis in ICU patients with high severity of disease: a post hoc analysis of the placebocontrolled SUP-ICU trial. Intensive Care Med. 2019;45(5):609-18.

5. Vieira LV, Pedrosa LAC, Souza VS, Paula CA, Rocha R. Incidence of diarrhea and associated risk factors in patients with traumatic brain injury and enteral nutrition. Metab Brain Dis. 2018;33(5):1755-60.

6. Reintam Blaser A, Jakob SM, Starkopf J. Gastrointestinal failure in the ICU. Curr Opin Crit Care. 2016;22(2):128-41

7. Vredebregt SJ, Moll HA, Smit FJ, Verhoeven JJ. Recognizing critically ill children with a modified pediatric early warning score at the emergency department, a feasibility study. Eur J Pediatr. 2019:178(2):229-34

8. Reintam A, Parm P, Kitus R, Starkopf J, Kern H. Gastrointestinal failure score in critically ill patients: a prospective observational study. Crit Care. 2008; 12(4):R90.
9. Bordejé ML, Montejo JC, Mateu ML, Solera M, Acosta JA, Juan M, GarcíaCórdoba F, García-Martínez MA, Gastaldo R. Intra-Abdominal Pressure as a Marker of Enteral Nutrition Intolerance in Critically III Patients. The PIANE Study. Nutrients. 2019;11(11):2616-25.

10. Agarwala R, Rana SS, Sharma R, Kang M, Gorsi U, Gupta R. Gastrointestinal Failure Is a Predictor of Poor Outcome in Patients with Acute Pancreatitis. Dig Dis Sci. 2020;65(8):2419-26.

11. Pan $P$, Song $Y$, Du X, Bai L, Hua X, Xiao Y, Yu X. Intestinal barrier dysfunction following traumatic brain injury. Neurol Sci. 2019;40(6):1105-10.

12. Kigerl KA, Mostacada K, Popovich PG. Gut Microbiota Are DiseaseModifying Factors After Traumatic Spinal Cord Injury. Neurotherapeutics. 2018;15(1):60-7.

13. Wang B, Wu G, Zhou Z, Dai Z, Sun Y, Ji Y, Li W, Wang W, Liu C, Han F, et al. Glutamine and intestinal barrier function. Amino Acids. 2015;47(10):2143-54

14. Wang DC, Bose P, Parmer R, Thompson FJ. Chronic intrathecal baclofen treatment and withdrawal: I. Changes in ankle torque and hind limb posture in normal rats. J Neurotrauma. 2002;19(7):875-86.

15. Midenfjord I, Polster A, Sjövall H, Törnblom H, Simrén M. Anxiety and depression in irritable bowel syndrome: Exploring the interaction with other symptoms and pathophysiology using multivariate analyses. Neurogastroenterol Motil. 2019:31(8):e13619.

16. Reintam Blaser A, Malbrain ML, Starkopf J, Fruhwald S, Jakob SM, De Waele J, Braun JP, Poeze M, Spies C. Gastrointestinal function in intensive care patients: terminology, definitions and management. Recommendations of the ESICM Working Group on Abdominal Problems. Intensive Care Med. 2012:38(3):384-94

17. Zhang D, Li Y, Ding L, Fu Y, Dong X, Li H. Prevalence and outcome of acute gastrointestinal injury in critically ill patients: A systematic review and metaanalysis. Med (Baltim). 2018;97(43):e12970.

18. Minhua C, Tao G, Fengchan X, Wenkui Y. Using Digestive Fluid Biomarkers to Predict Acute Gastrointestinal Injury in Critically III Patients: A Pilot Study. Am J Crit Care. 2018;27(6):504-7.

19. Ware AL, Shukla A, Goodrich-Hunsaker NJ, Lebel C, Wilde EA, Abildskov TJ, Bigler ED, Cohen DM, Mihalov LK, Bacevice A, et al. Post-acute white matter microstructure predicts post-acute and chronic post-concussive symptom severity following mild traumatic brain injury in children. Neuroimage Clin. 2020;25:102106

20. Perceval C, Szajewska H, Indrio F, Weizman Z, Vandenplas Y. Prophylactic use of probiotics for gastrointestinal disorders in children. Lancet Child Adolesc Health. 2019:3(9):655-62.

21. Wesselink E, Koekkoek K, Looijen M, van Blokland DA, Witkamp RF, van Zanten ARH. Associations of hyperosmolar medications administered via nasogastric or nasoduodenal tubes and feeding adequacy, food intolerance and gastrointestinal complications amongst critically ill patients: A retrospective study. Clin Nutr ESPEN. 2018;25:78-86.

22. Aperstein $Y$, Cohen L, Bendavid I, Cohen J, Grozovsky E, Rotem T, Singer $P$. Improved ICU mortality prediction based on SOFA scores and gastrointestinal parameters. PLoS One. 2019;14(9):e0222599.

23. Padar M, Starkopf J, Uusvel G, Reintam Blaser A. Gastrointestinal failure affects outcome of intensive care. J Crit Care. 2019;52:103-8.

24. Kansu A, Durmaz Ugurcan O, Arslan D, Unalp A, Celtik C, Sarıoglu AA. Highfibre enteral feeding results in improved anthropometrics and favourable gastrointestinal tolerance in malnourished children with growth failure. Acta Paediatr. 2018;107(6):1036-42.

25. Mak MHW, Low JK, Junnarkar SP, Huey TCW, Shelat VG. A prospective validation of Sepsis-3 guidelines in acute hepatobiliary sepsis: qSOFA lacks sensitivity and SIRS criteria lacks specificity (Cohort Study). Int J Surg. 2019; 72:71-7.

26. Liu Z, Meng Z, Li Y, Zhao J, Wu S, Gou S, Wu H. Prognostic accuracy of the serum lactate level, the SOFA score and the qSOFA score for mortality among adults with Sepsis. Scand J Trauma Resusc Emerg Med. 2019:27(1):51.

27. Pereira B, Dorigatti A, Melek M, Dos Santos J, Ferreira M, Calderan T, Carmona C, Fraga G. Septic shock patients admitted to the intensive care unit with higher SOFA score tend to have higher incidence of abdominal compartment syndrome - a preliminary analysis. Anaesthesiol Intensive Ther. 2019;51(5):370-2.

28. Lu XL, Oiu J, Zhu YM, Chen P, Zuo C, Tang L, Liu X, Xiao ZH, Du YK. [Role of Pediatric Critical IIIness Score in evaluating severity and prognosis of severe hand-foot-mouth disease]. Zhongguo Dang Dai Er Ke Za Zhi. 2015:17(9): $961-4$ 
29. Lu Y, Liu Z, Li S, Wang Y, Li C, Yuan E, Xing J, Guo W. [Nested case-contro study on paediatric early warning score and ventilator-associated complications in children with acute respiratory distress syndrome]. Zhong Nan Da Xue Xue Bao Yi Xue Ban. 2019:44(9):996-1002.

30. Lone NI, Lee R, Salisbury L, Donaghy E, Ramsay P, Rattray J, Walsh TS. Predicting risk of unplanned hospital readmission in survivors of critical illness: a population-level cohort study. Thorax. 2019;74(11):1046-54.

\section{Publisher's Note}

Springer Nature remains neutral with regard to jurisdictional claims in published maps and institutional affiliations.

Ready to submit your research? Choose BMC and benefit from:

- fast, convenient online submission

- thorough peer review by experienced researchers in your field

- rapid publication on acceptance

- support for research data, including large and complex data types

- gold Open Access which fosters wider collaboration and increased citations

- maximum visibility for your research: over $100 \mathrm{M}$ website views per year

At BMC, research is always in progress.

Learn more biomedcentral.com/submissions 